\section{International Scientific Journal Theoretical \& Applied Science}

p-ISSN: 2308-4944 (print) e-ISSN: 2409-0085 (online)

Year: $2014 \quad$ Issue: $11 \quad$ Volume: 19

Published: $30.11 .2014 \quad \underline{\text { http://www.T-Science.org }}$
Victor Aleksandrovich Melent'ev Philosophy Doctor, Senior research associate Institute of Semiconductor Physics Siberian Branch RAS, Russia melva@isp.nsc.ru

SECTION 4. Computer science, computer engineering and automation.

\title{
ABOUT TOPOLOGICAL COMPACTNESS OF COMPUTING SYSTEMS
}

Abstract: The problem of synthesis of topology of a computer system is solved as a problem of constructing a graph with a minimum diameter for given values of the order, degree and girth of the graph. The solution is based on the use of projective graph description and it is reduced to creation of a joint, in respect of the above characteristics, set of its vertex-complete projections. The concept of a compact graph is given, its analytical model is presented. A algorithm for generation compact graphs, which is based on this model, is described.

Key words: topology of communication networks of the computing systems CS, compactness of graphs of CS; diameter, order, degree and girth of the graph of CS.

Language: Russian

Citation: Melent'ev VA (2014) ABOUT TOPOLOGICAL COMPACTNESS OF COMPUTING SYSTEMS. ISJ Theoretical \& Applied Science 11 (19): 59-65. doi: http://dx.doi.org/10.15863/TAS.2014.11.19.12

\section{УДК 004.722.2+004.272.3}

\section{О ТОПОЛОГИЧЕСКОЙ КОМПАКТНОСТИ ВЫЧИСЛИТЕЛЬНЫХ СИСТЕМ 1}

Аннотация: Проблема синтеза топологии вычислительной системы решается как задача построения графа с минимальным диаметром при заданных значениях порядка, степени и обхвата графа. Решение основано на использовании проективного описания графа и сводится к построению совместной (в отношении указанных выше характеристик) системы его вериинно полных проекций. Дано понятие компактного графа, определена его аналитическая модель и изложен основанный на ней алгоритм генерации.

Ключевые слова: Топология сетей связи вычислительных систем (ВС), компактность графов ВС; диаметр, порядок, степень и обхват графа ВС.

\section{Введение.}

Масштабирование вычислительных и информационных ресурсов неразрывно связано с созданием и совершенствованием моделей, предоставляющих не только возможности синтеза топологии масштабируемых вычислительных систем (ВС) и сетей, но и возможности их использования для адекватного и безусловно актуализированного управления функционированием последних при сохранении ими функциональной и информационной целостности. Актуализированность управления заключается в том, что временной интервал от момента изменения состояния системы или отдельных ее элементов до момента завершения реагирования на эти изменения не должен превышать некоторого критического значения, определяюего временну́ю границу рациональности. К примеру, запаздывание реакции на разгрузку/перегрузку линий электропередачи может привести к потере устойчивости энергосистемы и к катастрофическим последствиям.

\footnotetext{
1 Работа выполнена при поддержке Российского фонда фундаментальных исследований (проект 14-07-00169a)
} 
Факторами запаздывания управляющей информации, приводящими к потере еe актуальности и последующему неэффективному, а в некоторых случаях и небезопасному управлению, могут быть: недостаточная оперативность подсистем мониторинга состояний системы и ее элементов, недостаточная эффективность алгоритмов интерпретации состояний и выработки управляющих воздействий и сетевые задержки в доставке как информации об изменении состояния, так и соответствующей этим изменениям управляющей информации. Задержки при этом наряду с качеством используемых в системе каналов и протоколов связи определяются ее топологией: наличие транзитов, характерное для неполносвязных систем, увеличивает пропорциональные числу транзитов задержки.

Оптимизация топологии ВС по критерию задержек позволяет высвободить дополнительные временны́е резервы для использования более качественных) алгоритмов интерпретации состояний и формирования управляющих воздействий. Таким образом, выбор топологии с минимальными задержками межмодульных взаимодействий является одним из важнейших факторов, определяющих управляемость и устойчивость системы. Добавим к этому особую критичность к времени реакции (следовательно, и к структуре) на конфигурационные изменения в отказоустойчивых ВС: промедление в локализации неисправностей может привести к каскадному их развитию и соответствующим катастрофическим последствиям и для самой системы, и для объектов ее управления.

Проблемы синтеза структур с заданными коммуникационными характеристиками традиционно решаются методами теории графов. При этом между множеством модулей системы и множеством вершин графа, а также между множеством линий связи и ребер графа устанавливают взаимно однозначные соответствия, а задержки в системах и сетях ассоциируют с метрическими характеристиками графов: диаметром, радиусом и эксцентриситетами вершин [1, с. 141]. Поэтому, анализируя здесь графы и оптимизируя их метрические характеристики, мы имеем в виду прежде всего структуры систем и сетей связи и оптимизацию соответствующих им коммуникационных свойств.

Известные исследования устойчивости систем к случайному и/или преднамеренному удалению вершин из описывающих их графов свидетельствуют о большей устойчивости регулярных структур, характеризуемых распределением степени вершин с не более чем тремя несовпадениями [2, с. 920]. Поэтому объектом исследования в данной работе являются регулярные графы ВС.
Наиболее распространенными методами решения проблемы синтеза структур с заданными свойствами являются стохастические методы, состоящие в генерации случайных графов с их фильтрацией по соответствующим заданным свойствам критериям [3 c. $4 ; 4$ с. 1] или основанные на генетических подходах. Однако в этом случае допустимо говорить лишь о вероятностнооптимальном синтезе, определяемом размером выборки или мощностью случайно сгенерированной первоначальной популяции, и недетерминированность результатов такого синтеза может привести к непредсказуемым деструктивным последствиям в процессе функционирования системы.

Возможности детерминированного синтеза структур ВС в настоящее время слабо изучены. Это связано с тем, что традиционно используемый аппарат анализа и преобразований графов основан на недостаточно формализованных и информационно емких описаниях. В работах [5 с. 696-698; 6 с. 167-171] впервые предложен способ описания графа скобочными его проекциями, изначально содержащими не только информацию о смежности вершин, но и об их достижимости, что исключает необходимость временны́х затрат на поиск кратчайших маршрутов; в работах [7 c. 213,8 с. 112] предложено использовать такие проекции в процессе оперативного управления, в частности, в процессах реконфигурации отказоустойчивых ВС. Аналитический подход к синтезу регулярных графов с заданными значениями порядка, степени и обхвата, основанный на представлении структуры проекциями графа ВС, впервые предложен в [9].

В данной работе в рамках задачи генерации регулярных структур ВС по критерию задержек введено понятие компактных структур (графов системы) и определены условия компактности. Предложен способ синтеза компактного графа с заданными значениями порядка и степени, основанный на построении базовой системы проекций суграфа, на совместном решении этой системы путем доопределения eе исходных проекций до реберной полноты.

\section{2. Основные положения.}

В данном разделе приведены некоторые используемые в работе определения и общие сведения о проективном описании графа.

Регулярный граф - связный граф $G(V, E)$, степени $\operatorname{deg}\left(v_{i}\right)=s_{i}$ всех вершин $v_{i} \in V$ которого равны между собой; при этом степень $s$ называется степенью $s(G)$ регулярного графа $G$. 
Эксиентриситет вершины - для данной вершины $u \in V \quad$ графа $G(V, E)$ величина $e(u)=\max _{u, v \in V} \partial(u, v)$, где $\partial(u, v)$ - расстояние между вершинами $u$ и $v$.

Диаметр - наибольшее расстояние между вершинами связного графа: $d(G)=\max _{u \in V} e(u)$.

Радиус графа - минимальный из эксцентриситетов вершин связного графа: $r(G)=\min _{u \in V} e(u)$.

Проекциия $P\left(v_{j}\right)$ графа $G(V, E)$ представляет собой многоуровневую конструкцию, на нулевом уровне которой расположена вершина $v_{j} \in V$, выбранная в качестве ракурсной; порожденное ею подмножество вершин первого уровня $V_{1 j} \subset V$ содержит все вершины ее окружения $\mathcal{N}\left(v_{j}\right)$, а $i$-й уровень $(i \geq 1)$ представляет собой совокупность подмножеств вершин, каждое из которых порождено вершиной $(i-1)$-го уровня и является окружением этой вершины без тех его вершин, что предшествуют ей в данной проекции. Таким образом, отношение «предшествования вершины/порождения подмножества» фактически моделирует отношение смежности предшествующей вершины вершинам порожденного ею подмножества. Формальная запись этих отношений в скобочном описании двух произвольно взятых соседних уровней проекции графа имеет вид

$$
v_{i 1} V_{i+1,1}, \ldots, v_{i j}{ }^{V_{i+1, j}},
$$

где вершины $v_{i 1}$ и $v_{i j}$ одного из подмножеств $i$-го уровня предшествуют и смежны вершинам порожденных ими подмножеств $V_{i+1,1}$ и $V_{i+1, j}$ вышестоящего $(i+1)$-го уровня. Технология построения скобочных описаний графа и их свойства достаточно подробно представлены в работах [5 с. 696-698; 6 с. 167-171] и обобщены в [9], поэтому здесь остановимся лишь на некоторых используемых в данной работе свойствах.

Вершине $v_{i j} k$-уровневой проекции $P_{k}\left(v_{0}\right)$, построенной из ракурсной вершины $v_{0}$, соответствует упорядоченное множество вершин $W\left(v_{i j}\right)=\left(v_{0}, v_{10}, \ldots, v_{i j}\right)$, представляющее собой простую цепь из $v_{0}$ в $v_{i j}$, длина этой цепи $\partial\left(v_{0}, v_{i j}\right)=i$. В общем случае некоторые (за исключением ракурсной) вершины проекции $P_{k}\left(v_{0}\right)$ могут быть $m_{i j}$-кратными: $0 \leq m_{i j} \leq \Sigma_{i} C_{i}-\Sigma_{i}\left|V_{i}\right|$, где $C_{i}$ - число элементов $i$-го уровня проекции $P_{k}\left(v_{0}\right)$, а $V_{i} \subset V-$ множество вершин графа, представленных $i$-м уровнем проекции. Отличие $m_{i j}$ от единицы означает наличие соответствующего числа простых цепей из ракурсной вершины $v_{0}$ в вершину $v_{i j}$.

В $[6$, с. 170] показано, что номер $i$ уровня в проекции $P\left(v_{0}\right)$ определяет удаленность вершин $V_{i}$ этого уровня от ракурсной вершины $v_{0}$ а также то, что уровень $k_{e}$, впервые доопределяющий множество вершин всех нижерасположенных уровней проекции графа $G(V, E)$ до $V$, соответствует эксцентриситету $e\left(v_{0}\right)$ ракурсной вершины $v_{0}$ в проекции $P\left(v_{0}\right)$ :

$$
e\left(v_{0}\right)=k_{e} \mid \cup_{i=0}^{k_{e}-1} V_{i} \subset V, \quad \mathrm{U}_{i=0}^{k_{e}} V_{i}=V .
$$

Назовем это условие условием вершинной полноты проекции. Однако реализации условия (1) в проекции не всегда достаточно для определения всех ребер описываемого ею графа. Проекция $P_{k}\left(v_{0}\right)$ графа $G(V, E)$ является полной, если ею определены все его вершины и ребра (отношения смежности). Необходимые условия полноты проекции могут быть записаны следующим образом:

$$
\bigcup_{i=0}^{k} V_{i}=V \text { и } \bigcup_{i=0}^{k} E_{i}=E,
$$

здесь $E_{i}=\left\{e_{u v} \mid u \in V_{i-1}, v \in V_{i}\right\}-$ множество ребер, инцидентных парам вершин соседних уровней проекции. Нетрудно заметить, что первое из этих условий (условие вершинной полноты) поглощается вторым (условием реберной полноты проекции).

Отметим также доказанное в [9, с. 77] свойство о числе уровней в полной проекции, согласно которому минимальное число уровней $k=k_{\min }\left(v_{0}\right)$ в проекции $P_{k}\left(v_{0}\right)$ связного простого графа $G(V, E)$, достаточное для полноты представления, не меньше эксцентриситета $e\left(v_{0}\right)$ ракурсной вершины $v_{0}$ и не больше увеличенного на единицу его значения:

$$
k_{\min }\left(v_{0}\right)=\left\{\begin{array}{cl}
e\left(v_{0}\right), & A(u, v)=0 \\
e\left(v_{0}\right)+1, & A(u, v)=1
\end{array}\right.
$$

Предикат $A(u, v)$ будет истинным, если в множестве $V_{e}$ вершин уровня рассматриваемой проекции с номером, равным эксцентриситету $e\left(v_{0}\right)$ ракурсной вершины $v_{0}$ этой проекции, найдется хотя бы две смежные вершины $u, v \in V_{e}, \partial(u, v)=1$, равноотстоящие от ракурсной вершины $v_{0}$ на величину еe эксцентриситета $e\left(v_{0}\right)$, т.е. $\exists\left\{u, v \in V_{k} \mid \partial\left(v_{0}, u\right)=\partial\left(v_{0}, v\right)=e\left(v_{0}\right)\right.$ и $\left.\partial(u, v)=1\right\}$.

Приведенные ниже и используемые далее леммы являются следствиями доказанного в [9, с. 78] утверждения, констатирующего, что обхват $g(G)$ графа $G(V, E)$, заданного проекцией $P\left(v_{0}\right)$, не превышает минимальной из сумм номеров ее уровней, содержащих одноименную вершину. Число повторений вершины $u \in V$ в проекции назовем еe кратностью и обозначим $m(u)$. Кратность $m(u)$ вершины $u$ в проекции и число описываемых ею простых цепей из ракурсной вершины $v$ в вершину $u$ равнозначны. Экземпляр $m$-кратной вершины, расположенный в проекции 
первым снизу и слева, будем называть ниже оригинальным, остальные экземпляры такой вершины, если они существуют $(m(u)>1)-$ реплицированными, или репликами.

Лемма 1. Если в проекции $P_{k}\left(v_{0}\right)$ графа $G(V, E)$ с числом уровней $k=e\left(v_{0}\right)$ кратность $m(u)$ вершины $u \in V$ больше единицы, то обхват графа $g(G)$ не превышает минимальной из сумм номеров уровней двух подмножеств, включающих в себя эту вершину:

$$
\begin{aligned}
& \exists u \in V\left(m_{u}>1\right) \Rightarrow g(G) \leq x_{1}+x_{2}, \text { где } \\
& x_{1}, x_{2} \in \vec{X}=\left(x_{1}, \ldots, x_{m_{u}}=i \mid V_{i j} \ni u, i=\left(\overrightarrow{1, e\left(v_{0}\right)}\right)\right) .
\end{aligned}
$$

Лемма 2. Если в проекции $P_{k}\left(v_{0}\right)$ графа $G$ с числом уровней $k=e\left(v_{0}\right)$ вершины с кратностью, большей единицы, отсутствуют $(\forall u \in V m(u)=1)$, то граничные значения обхвата $g(G)$ определены выражением $e\left(v_{0}\right)+2 \leq g(G) \leq 2 e\left(v_{0}\right)+1$.

\section{1. Компактные графы}

Максимально возможное при степени графа $s$ число вершин $C_{i}(s)$ отличного от нуля $i$-го уровня проекции $P\left(v_{0}\right), v_{0} \in V$, регулярного простого графа $G(V, E)$ определено выражением

$$
C_{i}(s)=s(s-1)^{i-1},
$$

а максимальное число вершин $N_{d}(s)$ регулярного графа, которое удастся поместить на $d$ уровнях проекции, соответствует максимальному порядку графа $G$ с диаметром $d$ :

$$
N_{d}(s)=1+s \sum_{i=1}^{d}(s-1)^{i-1} .
$$

Выше дано условие (1) вершинной полноты проекции графа - таким образом, синтез графа $G(V, E)$ порядка $n$ с диаметром $d(G)$ сводится к такому размещению вершин на каждой из $n$ проекций этого графа, что число уровней в проекциях, в соответствии с (1), не превысит этот диаметр. Из множества задач синтеза регулярных графов с заданными значениями порядка $n$ и степени $s$ вычленим задачи синтеза графов с минимально возможными при этих $n$ и $s$ диаметрами - такие графы далее будем называть $n(s)$-компактными.

Из (4) получим максимальное число вершин, которое может быть размещено на $d$-м и $(d-1)$-м уровнях проекции регулярного графа:

$$
\begin{gathered}
N_{d}(s)=1+s \sum_{i=1}^{d}(s-1)^{i-1}, \\
N_{d-1}(s)=1+s \sum_{i=1}^{d-1}(s-1)^{i-1} .
\end{gathered}
$$

Легко видеть, что первое из этих выражений определяет максимальный для заданного диаметра $d$ порядок $n$, тогда как во втором определен максимально возможный порядок графа с уменьшенным на единицу диаметром. Тогда условие компактности, корреспондирующее порядок и диаметр графа его степенью, имеет вид:

$$
1+s \sum_{i=1}^{d-1}(s-1)^{i-1}<n(s) \leq 1+s \sum_{i=1}^{d}(s-1)^{i-1} \text {. }
$$

Понятно, что в компактном $n(s)$-графе все вершины являются центральными, так как диаметр $d$ компактного графа минимален по определению, а наличие в таком графе вершин с эксцентриситетами, меньшими диаметра, исключено условием (5). Следовательно, и число уровней в любой вершинно-полной проекции такого графа не может превышать диаметра последнего. Понятно, если порядок $n(s)$-компактного графа равен верхнему граничному значению $N_{d}(s)$, то двух- или более кратные вершины в любой его проекции $P_{d}\left(v_{j}\right)$, $v_{j} \in V$, отсутствуют $\left(\forall u \in V m_{u}=1\right)$, и значения обхвата $g$ в соответствии с леммой 2 определяются из $d+2 \leq g(G) \leq 2 d+1$.

\section{2. Синтез компактных графов}

В $[9$, с. 74-86] впервые представлен аналитический подход к синтезу регулярных графов с заданными значениями порядка, степени, диаметра и обхвата. Для введенных здесь компактных графов последовательность действий по их синтезу дополнена.

1. Из (3) получим корреспондируемое заданными значениями порядка $n$ и степени/диаметра значение диаметра/степени компактного графа. Введем однозначную разметку $n$ вершин графа и выберем произвольную вершину в качестве ракурсной вершины базовой $d$ уровневой проекции не имеющего циклов остовного подграфа синтезируемого графа с заданными значениями порядка $n$, степени $s$ и диаметра $d$. Определим число тдвух- и более кратных вершин: $m=N_{d}(s)-n(s), \quad$ где $N_{d}(s)$ определим из (4). Если число $m$ равно нулю, то максимальный обхват $g$ (при реальности такого графа с этим значением $g$ ) в соответствии с леммой 3 составит $g=2 d+1$.

2 . Построим $d$-уровневую остовную проекцию $P^{\prime} d\left(v_{0}\right) \quad$ синтезируемого $n(s)$-компактного графа $G(V, E)$, выбрав в качестве ракурсной вершины $v_{0}$ любую из произвольным образом пронумерованных вершин, $v_{0} \in V$. Описываемый проекцией $P_{d}^{\prime}\left(v_{0}\right)$ граф $G^{\prime}\left(V, E^{\prime}\right) \subset G(V, E)$ является каркасом (остовным деревом) искомого графа с 
корнем в $v_{0}$. Он включает в себя все вершины графа с единичной кратностью $\left(m_{u}=1, u \in V\right)$ и не содержит циклов. Размещение $n$ вершин на $d$ уровнях этой проекции может быть произвольным, но таким, чтобы построенная проекция была вершинно-полной:

$\bigcup_{i=0}^{d} V_{i}=V$, или $\sum_{i=0}^{d}\left|V_{i}\right|=|V|$.

Вершины $V_{1}$, расположенные на первом уровне базовой проекции $P_{d}^{\prime}\left(v_{0}\right)$, являются окружением $\mathcal{N}\left(v_{0}\right)$ ракурсной вершины $v_{0}$, и их число $\left|V_{1}\right|$ равно степени $s$ синтезируемого графа; на остальных уровнях этой проекции число вершин $\left|V_{i}\right|$ при $2<i \leq d$ может быть меньшим, чем число $C_{i}(s)$ элементов этого уровня, определяемое из (3). В этом случае число $\left|V_{i+1}\right|$ вершин $(i+1)$-го уровня будет меньше числа $C_{i+1}(s)$, т.е. $\left|V_{i+1}\right| \leq\left|V_{i}\right| \cdot(s-1)$. Разность $C_{i}(s)-\left|V_{i}\right|$ равна числу неизвестных на $i$-м уровне проекции $P_{d}\left(v_{0}\right)$ элементов.

3. Соответствующие базовой проекции $P_{d}^{\prime}\left(v_{0}\right)$ окружения $\mathcal{N}^{\prime}\left(v_{j}\right)$ вершин сведем в список $\mathcal{N}^{\top}(G)=\left(\mathcal{N}^{\prime}\left(v_{j}\right) \mid v_{j} \in V\right)$

Вершины $v_{j} \in V$, окружения которых пока не определены полностью $\left(\left|\mathcal{N}^{\prime}\left(v_{j}\right)\right|<s\right)$, включим в множество $V^{\prime}=\left\{v_{j} \in V|| \mathcal{N}^{\prime}\left(v_{j}\right) \mid<s\right\} . \quad$ Окружения $\mathcal{N}^{\prime}\left(v_{j}\right) \quad$ этих вершин $\quad v_{j} \in V^{\prime} \quad$ дополняем потенциальными подмножествами $\mathcal{N}_{p}\left(v_{j}\right)=\left\{V^{\prime} \backslash\left\{W\left(v_{0}, v_{j}\right)\right\}_{x}\right\}$, нижний индекс $x$ при которых равен числу недостающих в этом окружении вершин, $\quad x=s-\left|\mathcal{N}\left(v_{j}\right)\right|$ : $\mathcal{N}\left(v_{j}\right)=\mathcal{N}\left(v_{j}\right) \cup \mathcal{N}_{p}\left(v_{j}\right)$; здесь $W\left(v_{0}, v_{j}\right)$ - множество всех предшественниц вершины $v_{j}$ в проекции $P^{\prime} d\left(v_{0}\right)$, составляющих простую цепь из $v_{0}$ в $v_{j}$.

Синтез $n(s)$-компактного графа при необходимости может быть обусловлен его обхватом, определяемым леммами 1 или 2. В этом случае потенциальные подмножества вершин $\mathcal{N}_{p}\left(v_{j}\right)$, входящие в состав $\mathcal{N}\left(v_{j}\right)$, должны быть соответствующим образом скорректированы: $\mathcal{N}_{p}\left(u_{i}\right):=$

$\mathcal{N}_{p}\left(u_{i}\right) \backslash\left\{v_{j} \in \mathcal{N}_{p}\left(u_{i}\right) \mid i, j \in\{1, \ldots, d\}, i+j<g\right\}, \quad-$ здесь индексы при вершинах $u_{i}, u_{j} \in V$ соответствуют номеру уровня проекции $P^{\prime} d\left(v_{0}\right)$, на котором эти вершины располагаются. Полученные таким образом окружения $\mathcal{N}^{\prime}\left(v_{j}\right)$ сведем в список $\mathcal{N}^{\top}(G)=\left(\mathcal{N}^{\top}\left(v_{j}\right) \mid v_{j} \in V\right)$.
4. Используя полученный в п. 3 список окружений $\mathcal{N}^{\top}(G)$ и учитывая (при необходимости) требуемое значение обхвата графа, поочередно выстраиваем остальные проекции $P_{d}\left(v_{j}\right), v_{j} \in V$, проводя каждый раз уточнение потенциальных подмножеств $\mathcal{N}_{p}\left(v_{j}\right)$ во вновь построенных проекциях и внося соответствующие изменения в список окружений $\mathcal{N}(G)$ графа и в построенные ранее проекции.

5. Задача синтеза графа будет решена, если список окружений его вершин не содержит потенциальных подмножеств:

$$
\forall v_{j} \in V\left(\left|\mathcal{N}\left(v_{j}\right)\right|=s,\left|\mathcal{N}_{p}\left(v_{j}\right)\right|=0\right) .
$$

Если в результате выполнения указанных действий для всех проекций синтезируемого графа хотя бы одно из окружений его вершин попрежнему является потенциальным (содержит потенциальное подмножество), то синтез $n(s)$ компактного графа не завершен, так как число вершин в построенном таким образом промежуточном варианте окружения не соответствует заданной степени. В этом случае в одном из потенциальных окружений следует выбрать вершину из его потенциального подмножества (произвести подстановку), скорректировав затем в соответствии с п. 4 все остальные проекции и список окружений вершин графа. Заметим при этом, что подстановки, не совместные с определенными заданием условиями, делают и систему проекций несовместной. Это выражается, в частности, в том, что мощности некоторых потенциальных подмножеств в отдельных проекциях системы становятся меньше числа вершин, неизвестных в соответствующих этим потенциальным подмножествам окружениях. В таком случае следует произвести возврат к предшествующей подстановке и выбрать альтернативный в данном потенциальном подмножестве вариант.

\section{3. Ограничения на обхваты в компактных графах}

Выше введено понятие $n(s)$-компактного графа, как регулярного графа $G(V, E)$ порядка $n$ и степени $s$, обладающего минимальным при заданных значениях $n$ и $s$ диаметром $d$. Представленное в общем виде условие компактности указывает на соответствующие диаметру $d$ и степени $s$ графа $G$ нижнюю и верхнюю границы его порядка:

$$
N_{d-1}<n \leq N_{d}
$$


Здесь верхний предел порядка $n$ определяется выражением (2), равен максимальному числу элементов в вершинно-полной проекции графа, при этом циклы с длиной, меньшей $2 d+1$, отсутствуют.

При наличии в графе циклов, с меньшей, чем $2 d+1$, длиной и учитывая связанное с этим появление реплик и уменьшение числа оригинальных вершин в вершинно-полной $d$ уровневой проекции при том, что число оригинальных вершин в ней равно порядку графа, запишем:

$$
N_{d-1}<n \leq N_{d}-r_{d}
$$

В $[11$, с. 19] показано, что для $n(s)$-компактного графа с четным обхватом справедливо

$$
\frac{s(s-1)^{d-1}-2}{s-2}<n \leq \frac{s(s-1)^{d-g / 2} \cdot\left((s-1)^{g / 2}-1\right)}{s-2},
$$

а для такого же графа с нечетным обхватом -

$$
\frac{s(s-1)^{d-1}-2}{s-2}<n \leq \frac{(s-1)^{d-\lfloor g / 2\rfloor} .\left(s(s-1)^{\lfloor g / 2\rfloor}-2\right)}{s-2} .
$$

Рассмотрим случаи, обусловливающие потенциальную возможность построения $n(s)$ компактного графа соотношениями между его диаметром $d$ и обхватом $g$ :

1) $g=2 d+1$. Из (7) получим:

$$
n_{d}=\frac{s(s-1)^{d}-2}{s-2} \text {. }
$$

Этот случай соответствует максимальному при данном соотношении $d$ и $g$ порядку $n(s)$-компактного графа: сумма номеров уровней оригинальной вершины и ее реплики не может быть меньшей обхвата, поэтому уровни $i \leq d$ не содержат реплик, и число оригинальных вершин в вершинно-полной $d$-уровневой проекции максимально. По этой же причине $(d+1)$-й уровень каждой проекции включает в себя только реплики вершин исключительно $d$-го уровня. Число реплик $N(d+1)=s(s-1)^{d}$ превышает число $N(d)=s(s-1)^{d-1}$ оригинальных вершин в $(s-1)$ раз, - это указывает на то, что хорды остовного каркаса $T\left(v_{0}\right)$ графа, замыкают между собой только вершины $d$-го уровня. Таким образом, все циклы фундаментальной системы, ассоциированной с любой из проекций графа, обладают одинаковой, равной его обхвату $g=2 d+1$, нечетной длиной. Из (7) видно, что порядок такого графа, - если он существует, - имеет единственно возможное значение.

2) $g=2 d$. Из (6) получим:

$$
\frac{s(s-1)^{d-1}-2}{s-2}<n \leq \frac{s(s-1)^{d}-2}{s-2} \text {. }
$$

3) $g<2 d$ : $g=2 d-x, \quad 1 \leq x \leq 2 d-3$. Максимальному значению переменной $x=2 d-3$ соответствует наличие в графе 3-цикла: $g=2 d-$ $x=3$. Полученные из (6) и (7) условия компактности имеют вид:

$$
\begin{aligned}
& \text { для четных обхватов } g- \\
& \qquad \frac{s(s-1)^{d-1}-2}{s-2}<n \leq \frac{s(s-1)^{x / 2}\left((s-1)^{d-x / 2}-1\right)}{s-2} ;
\end{aligned}
$$

для нечетных $g$ -

$$
\frac{s(s-1)^{d-1}-2}{s-2}<n \leq \frac{(s-1)^{(x+1) / 2}\left(s(s-1)^{d-(x+1) / 2}-2\right)}{s-2}
$$

\section{4. Заключение}

В рамках проблемы повышения устойчивости функционирования больших систем путем снижения временны́х затрат на доставку контрольно-диагностической и управляющей информации и высвобождения временны́х резервов для реализации более качественных управляющих процедур и алгоритмов в статье решается задача синтеза регулярных структур ВС, обладающих минимальными задержками при заданных значениях порядка и степени представляющего систему графа.

Задача синтеза регулярной ВС с заданными значениями порядка, степени при минимуме диаметра еe графа сведена к построению совместной в отношении заданных свойств системы вершинно-полных проекций графа ВС и ее решению. Дано формальное изложение последовательности действий в процессе синтеза.

Выявлена формальная связь диаметра и числа вершин компактного графа с его обхватом. Это позволяет изначально исключить из потенциальных подмножеств искомых вершин те из них, которые могли бы войти в состав циклов с недопустимой длиной, что упрощает систему проекций генерируемого графа и ее решение в процессе генерации структур.

\section{References:}

1. Rapoport AM (2005) Metricheskie harakteristiki grafov setej kommunikatsij. Trudy ISA RAN 2005. - T. 14. - pp. 141-147.
2. Lakamraju V, Koren Z, Koren I, Krishna M (1998) Measuring the vulnerability of interconnection networks in embedded systems. 
Proc. First Merged Symp. IPPS/SPDP, EHPC Workshop, April 1998, pp. 919-924.

3. Donetti L, Hurtado PI, Mufioz MA (2005) Entangled networks, synchronization and optimal network topology. Phys. Rev. Lett. 95, 188701.

4. Weinstein, Eric W (2010) Cage Graph. http://mathworld.wolfram.com/topics/CageGraph s.html (17.07.10).

5. Melentiev VA (2004) Formalnye osnovy skobochnyh obrazov $\mathrm{v}$ teorii grafov. Trudy II Mezhdunarodnoj konferentsii "Parallelnyie vychisleniya i zadachi upravleniya" PACO'2004: In-t problem upravleniya RAN im. V.A. Trapeznikova, pp. 694-706.

6. Melentiev VA (2005) Formalnyj podhod k issledovaniyu struktur vychislitelnyh sistem. Vestnik Tomskogo gosudarstvennogo universiteta. No.14, pp. 167-172.

7. Melentiev VA (2006) Operatsii nad proektsiyami grafov i aktualizatsiya opisanij otkazoustoychivyh sistem. Vestnik Tomskogo gosudarstvennogo universiteta. \#17, pp. 208-213.

8. Melentiev VA (2008) Poisk vershinnyih (s,t)sechenij grafa vychislitelnoj sistemy $\mathrm{s}$ ogranicheniem po diametru komponent svyaznosti. Prikladnaya diskretnaya matematika, \#2(2), pp. 107-112.

9. Melentiev VA (2010) Analiticheskij podhod k sintezu regulyarnyh grafov $\mathrm{s}$ zadannymi znacheniyami poryadka, stepeni i obhvata. Prikladnaya diskretnaya matematika. \# 2(8), pp. 74-86.

10. Melentiev VA (2011) Kompaktnye struktury vychislitelnyh sistem $i$ ih sintez. Upravlenie bolshimi sistemami. Vyp. \# 32, pp. 107-112.

11. Melentiev VA (2012) Metrika, tsiklomatika i sintez topologii sistem i setej svyazi. Trudy Shestoj mezhd. konf. «Parallelnye vychisleniya i zadachi upravleniya» PACO'2012, Moscow, 2426 oct. 2012. IPU RAN, T. 3, pp. 10-25. 